\title{
Alcohol consumption and incidence of prostate cancer among Chinese people: A systematic review and meta-analysis
}

\author{
Jinhui Zhao*1,2 $\mathrm{PhD}$, Di Gao*3, MD, Yanhui $\mathrm{Li}^{3}, \mathrm{MD}$, Tim Stockwell ${ }^{1,4}, \mathrm{PhD}$ and Jun Ma, $\mathrm{PhD}^{3}$ \\ ${ }^{1}$ Canadian Institute for Substance Use Research (CISUR), University of Victoria, BC, Canada \\ ${ }^{2}$ School of Public Health, Soochow University, Suzhou, Jiangsu, China \\ ${ }^{3}$ School of Public Health \& Institute of Child and Adolescent Health, Peking University, China \\ ${ }^{4}$ Department of Psychology, University of Victoria, BC, Canada \\ * Joint first authors
}

\begin{abstract}
Aims: Meta-analyses have suggested a dose-response relationship between level of alcohol use and risk of prostate cancer, but the populations in the included studies are predominantly Caucasian. Many Chinese language studies have not been included in published reviews and/or meta-analyses. The present meta-analysis accessed research reports in both English and Chinese language sources in order to investigate this relationship specifically among Chinese people.

Methods: Searches in five large Chinese biomedical bibliographic databases were made for case-control and cohort studies of alcohol consumption and prostate cancer incidence and death (ICD-10: C61) up to May 2017. Studies were coded for design, outcome, drinker and non-drinkers, extent of control for confounding and other study characteristics. Mixed models were used to estimate relative risk (RR) of incidence or death from prostate cancer due to alcohol consumption with study level controls for designs, drinker bias and types of drinkers.
\end{abstract}

Findings: A total of 415 studies were identified of which 25 (20 in Chinese from five Chinese databases and 5 in English from published meta-analyses) satisfied inclusion criteria providing 36 risk estimates of prostate cancer for drinkers versus non-drinkers. There was a total of 36 OR estimates; 27 using patients as controls and 9 using healthy people. Nine studies (14 OR estimates) specified reference abstainers as "never drank" or "no drinking". Adjusted RR estimates indicated a significantly increased risk of prostate cancer among drinkers $(\mathrm{RR}=1.46$, 95\% CI: 1.40 - 1.52, t-test $\mathrm{P}<0.001)$ compared to non-drinkers. Dose-response relationships ( $\mathrm{t}$-test $\mathrm{P}<0.001$ ) were evident in three studies that assessed level of alcohol intake.

Conclusions: There is a significantly higher risk of prostate cancer incidence among Chinese drinkers than non-drinkers, with some evidence of a dose-response relationship. However, almost all the identified studies suffered from former and/or occasional drinker biases. Few studies had adequate measures of level of alcohol intake and further well-designed studies are required.

Prostate cancer is the development of cancer in the prostate, a walnut-sized gland in men that surrounds the top of the urethra and which produces seminal fluid (Bostwick, Crawford, Higano, \& Roach, 2004). Its growth and functions are controlled by male hormones such as testosterone. Prostate cancer is the second most common cancer in men worldwide. Around 1.1 million cases were recorded in 2012, accounting for $15 \%$ of all new cases of cancer in men (Forman \& Ferlay, 2014). It is the fifth most common cause of cancer death in men worldwide. There have been marked geographic, racial and ethnic variations in incidence and mortality of prostate cancer variously due to underdiagnosis, underreporting, differences in screening practices, differences in health-care access, gaps in knowledge and awareness and prevalence level of risk factors such as alcohol consumption (Hsing \& Devesa, 2001; Taitt, 2018). It is most commonly diagnosed in high-income western countries, where screening is common. However, the incidence of prostate cancer appears to be increasing in Asian countries such as Japan and China even though prostate-specific antigen (PSA) testing is not often used (Baade, Youlden, \& Krnjacki, 2009). It has been recognized

Correspondence: Dr. Jinhui Zhao, Canadian Institute for Substance Use Research (CISUR), University Victoria, PO Box 1700 STN CSC, Victoria, BC V8Y 2E4, Canada.

Email: zhaoj@uvic.ca

Financial support: Canadian Institute for Substance Use Research (CISUR) of University of Victoria Endowment Fund; Institute of Child and Adolescent Health of Peking University.

Declaration of interest: None.

Keywords: prostate cancer, alcohol, meta-analysis, mixed model, Chinese population. 
that prostate cancer is a currently common disease among Chinese males. The incidence rate was about 9.8 per 100,000 males in 2014, a significantly increased trend since 2008 (National Health Commission of the People's Republic of China, 2019; Ye \& Zhu, 2015). Therefore, prostate cancer as a chronic disease has become an important public health concern in developing countries such as China.

The risk factors for prostate cancer that can be considered established include age, race/ethnicity and family history (Gann, 2002). Many observational studies conducted mainly in Caucasian populations have investigated alcohol consumption as a risk factor for prostate cancer. Conclusions from these studies and of reviews have been conflicting with some finding increased risk of prostate cancer (Hayes et al., 1996; Sesso, Paffenbarger, \& Lee, 2001; Watters, Park, Hollenbeck, Schatzkin, \& Albanes, 2010), or decreased risk (Dagnelie, Schuurman, Goldbohm, \& Van den Brandt, 2004) and others finding no relationship (Hiatt, Armstrong, Klatsky, \& Sidney, 1994; Longnecker, 1995; Morton, Griffiths, \& Blacklock, 1996; Stemmermann, Nomura, Chyou, \& Yoshizawa, 1990; Tavani, Negri, Franceschi, Talamini, \& Lavecchia, 1994; Vandergulden, Verbeek, \& Kolk, 1994). Over the past few decades there have been several reviews and meta-analyses conducted to examine the association of prostate cancer with alcohol consumption (Bagnardi, Blangiardo, La Vecchia, \& Corrao, 2001; Breslow \& Weed, 1998; Dagnelie et al., 2004; Dennis, 2000; Fillmore, Chikritzhs, Stockwell, Bostrom, \& Pascal, 2009; Li, Yang, \& Cao, 2011; Longnecker, 1995; Morton et al., 1996; Rota et al., 2012; Zhao, Stockwell, Roemer, \& Chikritzhs, 2016) because of inconsistent results regarding the relationship between prostate cancer and alcohol consumption across individual studies. The most recent one, mainly based on the studies conducted in western countries shows a significant dose-response relationship (Zhao et al., 2016).

Because prior reviews and/or meta-analyses were conducted based on the studies searched and analyzed in English journals from English databases, these studies were mainly conducted with Caucasian populations. These published reviews and/or meta-analyses included few studies conducted in non-Caucasian populations such as Asians. Few studies conducted in Asian populations have been published in English journals and thus included in published reviews and meta-analyses. Over years, only six studies conducted on the Chinese population in China were included in published reviews and meta-analyses and one of these six studies was published in a Chinese journal (Breslow \& Weed, 1998; Dennis, 2000; Wei, Tang, Yang, Zhan, \& Yin, 1994) and other five in English journals (Hsing et al., 1994; Jian, Xie, Lee, \& Binns, 2004; Lee et al., 1998; Li et al., 2008; Yang et al., 2006). Meta-analysis by Li et al. (2011) included only four case-control studies of prostate cancer and alcohol consumption and found no relationship (Li et al., 2011). Among these six reviewed studies (nine risk estimates) in Chinese populations living in mainland China, only one shows a significantly increased risk when healthy people were treated as controls (Hsing et al., 1994). No studies were conducted on Chinese populations in other countries or regions. It is unclear whether or not those studies published in Chinese journals or non-English journals that cannot be located in English databases show different results as the risk estimates in published metaanalyses. Biological studies suggested that the effects of alcohol use on incidence of prostate cancer differed by race and/or environment because encoded enzymes involved in metabolizing alcohol differ by race. Alcohol is metabolized in the body mainly by the liver. Most of the ethanol in the body is broken down in the liver by an enzyme called alcohol dehydrogenase (ADH) which transforms ethanol into a toxic compound called acetaldehyde, a known carcinogen (Edenberg, 2007). Acetaldehyde is quickly broken down to a less toxic compound called acetate (Edenberg, 2007) by another enzyme called acetate dehydrogenase (ALDH). Acetate is then broken down to carbon dioxide and water for easy elimination (Edenberg, 2007). A person's risk of alcohol-related cancers is influenced by their genes that encode enzymes involved in metabolizing alcohol (DruesnePecollo et al., 2009). Many East Asian people carry a version of the gene for ADH that codes for a "superactive" form of the enzyme. This superactive ADH enzyme speeds the conversion of alcohol to toxic acetaldehyde. Among people of Japanese descent, those who have this form of ADH have a higher risk of pancreatic cancer than those with the more common form of ADH (Kanda et al., 2009). Research finds that genes encoding enzymes such as ADH1B involved in alcohol metabolism are polymorphic and that the superactive $\mathrm{ADH} 1 \mathrm{~B} * 2$ allele is highly prevalent $(54-96 \%)$ among East Asians (Goedde et al., 1992) but relatively rare among Caucasians $(1-23 \%)$. The less active ADH1B*1 is a risk factor for alcoholism in both Asians and Caucasians (Zintzaras, 2006; Zintzaras, Stefanidis, Santos, \& Vidal, 2006). Some people of East Asian descent carry a variant of the gene for ALDH2 that encodes a defective form of the enzyme. In people who produce the defective enzyme, acetaldehyde builds up when they drink alcohol. The accumulation of acetaldehyde has such unpleasant effects that most people who have inherited the ALDH2 variant are unable to consume large amounts of alcohol and therefore have a low risk of developing alcohol-related cancers such as prostate cancer. Genetic differences in these enzymes suggest that the effect of alcohol consumption on prostate cancer maybe different among Asians such as Chinese from that found among Caucasians and it is thus necessary to investigate the relationship between prostate cancer and alcohol consumption in non-Caucasians, for instance in Chinese populations.

There has been a marked increase in the numbers of cancers diagnosed in China between 2000 and 2011 (Chen et al., 2016). Much of this is explained by the aging and growth of the population. Other factors that may have contributed to the increase in the burden of cancer include increases in the prevalence of unhealthy behaviors or cancer-related lifestyle and improvements in disease awareness, detection services, and data completeness. The largest increase in incidence was seen for cancers of the prostate for men and cervix and thyroid for women. The factors driving the increase in prostate cancer are not entirely understood. However, they may include gradual implementation of PSA screening and improved biopsy techniques (Ito, 2014) or the impact of an increasingly westernized lifestyle including alcohol 
consumption (Baade, Youlden, Cramb, Dunn, \& Gardiner, 2013; Baade et al., 2009). China has a large population that accounts for approximately $20 \%$ of world population (United Nations Department of Economic and Social Affairs Population Division, 2017) and thus the increasing number of prostate cancer cases is a significant and growing proportion of the total globally. There has not been largescale screening for prostate cancer diagnosis in China (National Health Commission of the People's Republic of China, 2019). Alcohol consumption as a risk factor of many cancers has been increasing in China over years; per capita ethanol per male adult aged 15+ increased to 11.19 litres in 2017 from 7.08 in 1990 and 11.00 in 2010 (Manthey et al., 2019). The objective of the present meta-analysis was to investigate the relationship between prostate cancer and alcohol consumption among Chinese people.

\section{Methods}

\section{Inclusion and exclusion criteria}

The criteria for inclusion were: (i) case-control and cohort studies evaluating the relationship between alcohol consumption and incidence or death from prostate cancer; (ii) original articles published in Chinese Journals (Mandarin) or English Journals (English) up till April 2017; (iii) articles that reported findings as an odds ratio, hazard ratio, incidence ratio or standardized mortality ratio; (iv) studies conducted among Chinese people living in Mainland China; (v) articles reporting at any levels of alcohol consumption with drinking amounts, including the reference level; and (vi) studies reporting total alcohol consumption were included while studies based on consumption of specific beverages only such as wine, whiskey, vodka, sake or hard liquors were excluded. When the results of the study were published more than once or if the same dataset was used multiple times, only the most recent or more complete data were included in analyses. When published studies and dissertations were based on the same studies, the published articles were included in the analysis. When published studies used the data from the same studies, the studies that used the large samples were included. The primary outcomes of interest were mortality and/or morbidity from prostate cancer (ICD-9: 185 or ICD-10: C61) (WHO, 2010).

\section{Search strategy}

The systematic review follows the Preferred Reporting Items for Systematic Reviews and Meta-Analyses (PRISMA) guidelines (Moher, Liberati, Tetzlaff, Altman, \& the PRISMA Group, 2009). The study identified all potentially relevant articles published in Chinese Journals by searching five large Chinese biomedical bibliographic databases including China National Knowledge Infrastructure (CHKD-CNKI), Chinese Medical Current Content (CMCC), Chinese Biomedical Literature Database (CBM), VIP information/Chinese Scientific Journal Database (csjdvip,VIP database) and Wan-Fang Database/Chinese Medicine Premier. The combined five databases cover all studies conducted in China and published in Mandarin journals (Xia, Wright, \& Adams, 2008). The relevant articles were also searched through reference list cross-checking including those of previous meta-analyses (mainly English articles) and incorporating publications up to April 31, 2017. Hand searches of cited references in the selected articles, reviews and meta-analysis published on the same topic were also performed. The following MESH terms and text words were used: ("prostatic neoplasms" OR ("prostate" AND "neoplasms") OR "prostate cancer "OR ("prostate" AND "Cancer")) AND ("alcohol" OR (alcohol drinking) OR "alcohol consumption" OR "alcohol intake" OR ("alcohol" AND “consumption”)).

\section{Study selection}

Two reviewers (DG, YL) read the titles and/or abstracts of all the citations retrieved from the electronic database searches and removed all citations that were clearly not related to studies of the relationship between prostate cancer and alcohol consumption. Full-text articles that met eligibility criteria were obtained for data collection. Two of the authors (DG, YL) independently evaluated all studies selected for inclusion. The initial search identified a total of 415 studies of which 21 studies (Dong et al., 2016; Jia et al., 2013; Liang, Liu, Zhou, Gao, \& Chen, 2007; Liu, Wang, Gu, \& Zhou, 2014; Liu, J. et al., 2014; Lu, Hua, \& Wang, 2011; Ma, 2014; Min, Mi, Shao, Feng, \& Hua, 2012; Shao et al., 2012; Wang et al., 2014; Wei, Bin, \& Huang, 2017; Wei et al., 1994; Wu, Xu, \& Li, 2010; Wu, 2011; Wu, 2008; Xu, 2010; Xu et al., 2007; Yang, 2006; Zhang, Qiu, Shi, Liu, \& Chou, 1999; Zhang, Liang, \& Cui, 2012; Zhang et al., 2011) satisfied the criteria for the meta-analysis after removing 394 records for reasons identified in Figure 1. In addition we also included seven risk estimates from five studies (four published in English and one in Chinese journal) included in previously published reviews and meta-analyses (Hsing et al., 1994; Jian et al., 2004; Lee et al., 1998; Li et al., 2008; Yang et al., 2006). The study by Yang et al. (2006) published in an English journal is the same study as the thesis by Yang (2006) and the article by Yang et al (2009) (Li et al., 2011; Yang et al., 2009). Our study thus includes the study published in English journal because of the large sample size of cases. This meta-analysis includes 25 original studies in which 36 ORs are presented. All 25 included studies are case-control studies which examined the relationship between incidence of newly-diagnosed prostate cancer and alcohol consumption. No cohort studies on the topic conducted in Chinese population were identified.

\section{Data extraction}

Two reviewers (DG, YL) independently reviewed all eligible papers to extract and code data from all studies fulfilling the inclusion criteria, and any disagreements were resolved by discussion. Where disagreements existed, both reviewers discussed the discrepancy until a consensus was reached. Each study was coded with reference to a standardized code-book created and used in several published meta-analyses (Stockwell et al., 2016; Zhao et al., 2016; Zhao, Stockwell, Roemer, Naimi, \& Chikritzhs, 2017) (available from authors on request). The coding of all variables in the meta-dataset was double-checked by two other authors (JZ, TS). The data to be extracted were: (1) 
outcome, mortality or morbidity of prostate cancer and risk estimates; (2) types of study design and types of control used in case-control studies; (3) measures of alcohol consumption; (4) study characteristics; and (5) controlled variables in individual studies.

\section{Figure 1}

Flow diagram of systematic review of studies examining the relationship between alcohol consumption and prostate cancer among Chinese people

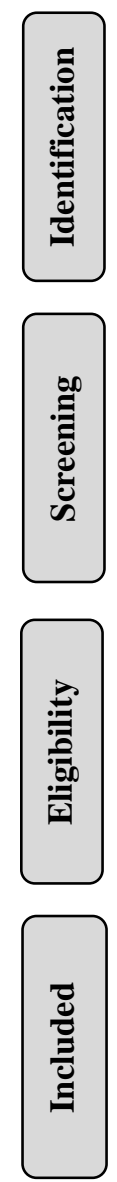

The study used various controls (shown in Table 1) and we examined the effect of types of control. These included studies defined "drinkers" and "non-drinkers" variously (shown in Table 2). No studies investigated the doseresponse relationship of prostate cancer and alcohol consumption and thus we coded definitions of alcohol drinkers and non-drinkers in each study and assessed whether former and occasional or light drinkers were misclassified into the reference group consisting of lifetime non-drinkers.

\section{Strategy for data analysis}

All included studies used case-control designs and thus the odds ratio (OR) estimates in case-control studies were entered as observations of the estimated risk relationships for meta-analysis. Mixed effects models were used to obtain the summarized risk estimates (Woodward, 2000).

In mixed multivariate meta-regression analysis, the dependent variable was the natural $\log$ of the RR estimated using the ORs of prostate cancer for drinkers and nondrinkers. Sensitivity analyses were run after excluding the studies by one by one and excluding the studies published in English journals and included in previously published metaanalyses. An analysis on dose-response relationship was performed. More details of statistical analysis strategy can be found in Appendix I. All significance tests assumed twotailed $\mathrm{P}$ values or $95 \%$ CIs. All statistical analyses were performed using SAS 9.4. 
Table 1

25 case-controlled studies with 36 odds ratio (OR) estimates and the corresponding $95 \%$ confidence interval (CI) selected for analysis of the association of incidence of prostate cancer with alcohol consumption among Chinese people, 1994-2017

\begin{tabular}{|c|c|c|c|c|c|c|c|}
\hline Author & Pub year & $\begin{array}{l}\text { No. of } \\
\text { cases }\end{array}$ & $\begin{array}{c}\begin{array}{c}\text { No of } \\
\text { controls }\end{array} \\
\end{array}$ & Mean age & $\begin{array}{c}\text { Control } \\
+ \\
\end{array}$ & OR $(95 \% \mathrm{CI})$ & $\begin{array}{c}\text { Covariate assessed } \\
\ddagger\end{array}$ \\
\hline Hsing et al. & 1994 & 135 & 314 & 68.93 & 5 & $1.56(1.02-2.38)$ & $1,2,3$ \\
\hline Hsing et al. & 1994 & 135 & 158 & 68.93 & 1 & $0.67(0.40-1.11)$ & $1,2,3$ \\
\hline Hsing et al. & 1994 & 135 & 157 & 69.08 & 3 & $0.78(0.47-1.29)$ & $1,2,3$ \\
\hline Wei et al. & 1994 & 27 & 54 & & 3 & $1.00(0.41-2.40)$ & $1,2,3,4$ \\
\hline Lee et al. & 1998 & 133 & 265 & 69.50 & 1 & $1.53(0.99-2.36)$ & $1,2,3,4$ \\
\hline Zhang et al. & 1999 & 137 & 137 & 69.39 & 7 & $2.04(1.26-3.29)$ & $1,2,3$ \\
\hline Jian et al. & 2004 & 88 & 212 & 71.87 & 5 & $1.29(0.78-2.11)$ & $1,2,3,4$ \\
\hline Jian et al. & 2004 & 92 & 204 & 71.87 & 5 & $1.22(0.72-2.06)$ & $1,2,3$ \\
\hline Yang et al. & 2006 & 225 & 250 & 71.28 & 4 & $1.15(0.76-1.74)$ & $1,2,3$ \\
\hline Liang et al. & 2007 & 50 & 58 & 69.32 & 2 & $1.40(0.35-5.56)$ & $1,2,3,4$ \\
\hline Liang et al. & 2007 & 56 & 57 & 69.32 & 7 & $1.33(0.52-3.39)$ & $1,2,3,4$ \\
\hline Liang et al. & 2007 & 50 & 56 & 69.32 & 2 & $1.99(0.38-10.54)$ & $1,2,3,4$ \\
\hline Liang et al. & 2007 & 56 & 57 & 69.32 & 7 & $2.17(0.71-6.64)$ & $1,2,3,4$ \\
\hline $\mathrm{Xu}$ et al. & 2007 & 207 & 235 & 71.20 & 5 & $1.62(1.03-2.56)$ & $1,2,3$ \\
\hline Li et al. & 2008 & 28 & 280 & 68.00 & 1 & $1.03(0.47-2.24)$ & $1,2,3,4$ \\
\hline Wu et al. & 2008 & 121 & 127 & 70.86 & 2 & $1.29(0.69-2.43)$ & $1,2,3,4$ \\
\hline Wu et al. & 2008 & 117 & 121 & 70.86 & 7 & $1.53(0.70-3.32)$ & $1,2,3,4$ \\
\hline Wu et al. & 2008 & 121 & 126 & 70.86 & 2 & $1.71(0.86-3.40)$ & $1,2,3,4$ \\
\hline Wu et al. & 2008 & 117 & 125 & 70.86 & 7 & $1.53(0.75-3.10)$ & $1,2,3,4$ \\
\hline Wu et al. & 2010 & 209 & 268 & 71.56 & 5 & $0.82(0.57-1.18)$ & $1,2,3$ \\
\hline Xu et al. & 2010 & 141 & 282 & 71.11 & 5 & $2.06(1.06-3.95)$ & $1,2,3$ \\
\hline Lu et al. & 2011 & 200 & 279 & 71.56 & 1 & $0.80(0.56-1.16)$ & $1,2,3,4$ \\
\hline Wu et al. & 2011 & 434 & 1,531 & 74.88 & 1 & $1.53(0.50-4.35)$ & $1,2,3,4$ \\
\hline Zhang et al. & 2011 & 108 & 242 & 70.01 & 1 & $1.63(1.03-2.57)$ & $1,2,3,4$ \\
\hline Min et al. & 2012 & 168 & 208 & 69.00 & 1 & $2.01(1.27-3.18)$ & $1,2,3,4$ \\
\hline Shao et al. & 2012 & 174 & 252 & 73.77 & 1 & $1.84(1.19-2.84)$ & $1,2,3,4$ \\
\hline Zhang et al. & 2012 & 60 & 40 & 75.85 & 2 & $2.46(1.02-5.91)$ & $1,2,3,4$ \\
\hline Jia et al. & 2013 & 86 & 86 & 72.22 & 2 & $1.27(0.64-2.50)$ & $1,2,3,4$ \\
\hline Jia et al. & 2013 & 86 & 86 & 72.22 & 7 & $1.94(0.94-4.01)$ & $1,2,3,4$ \\
\hline Liu et al. & 2014 & 267 & 534 & 70.84 & 2 & $1.54(1.13-2.10)$ & $1,2,3,4$ \\
\hline Liu et al. & 2014 & 120 & 120 & 73.12 & 7 & $1.44(0.82-2.54)$ & $1,2,3,4$ \\
\hline Liu et al. & 2014 & 120 & 120 & 73.12 & 2 & $1.58(0.89-2.80)$ & $1,2,3,4$ \\
\hline Ma et al. & 2014 & 103 & 100 & 71.20 & 5 & $0.85(0.31-2.30)$ & $1,2,3$ \\
\hline Wang et al. & 2014 & 410 & 440 & 71.40 & 1 & $1.36(1.01-1.84)$ & $1,2,3$ \\
\hline Dong et al. & 2016 & 664 & 702 & 71.50 & 6 & $1.30(1.02-1.65)$ & $1,2,3,4$ \\
\hline Wei et al. & 2017 & 128 & 128 & 71.56 & 5 & $2.23(1.24-3.99)$ & $1,2,3$ \\
\hline
\end{tabular}

$\dagger 1=$ healthy people; $2=$ benign prostatic hyperplasia; $3=$ other cancer; $4=$ other urological disease; $5=$ =patients without cancer, $6=$ patients without prostate cancer, $7=$ patients without prostate disease.

† Controlled confounders: $1=$ age; $2=$ race; $3=$ residence; $4=$ smoker. 
Figure 2

36 odds ratio (95\% CI) estimates of prostate cancer incidence for any alcohol consumption versus abstaining in 25 Chinese case-controlled studies

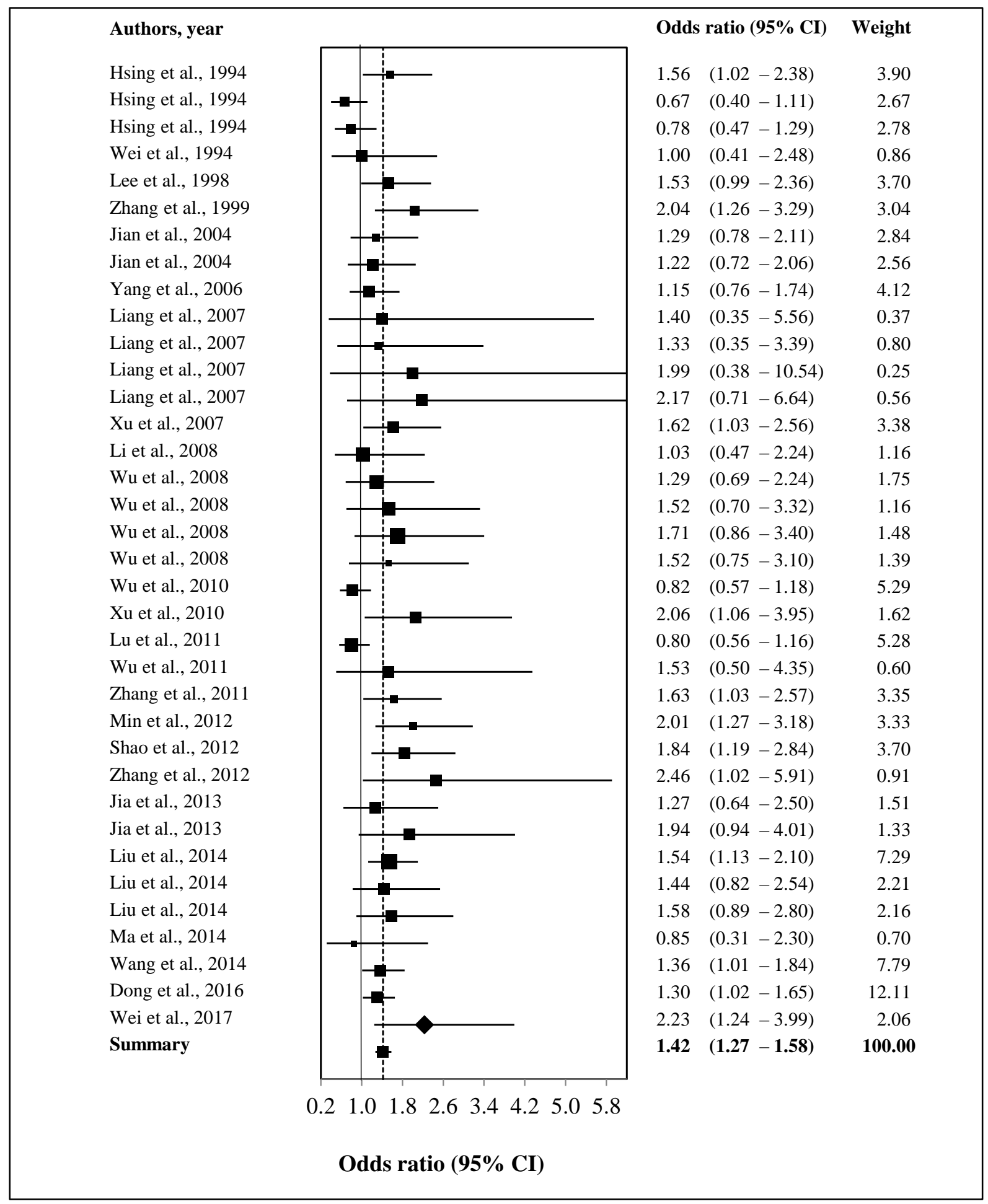




\section{Results}

There were 25 case-control studies identified spanning the years 1994-2017 that examined the relationship between prostate cancer incidence and alcohol consumption; four of them are dissertations in Chinese (Ma, 2014; $\mathrm{Wu}, 2011 ; \mathrm{Wu}$, 2008; Xu, 2010) and 16 studies were published in Chinese journals (Dong et al., 2016; Jia et al., 2013; Liang et al., 2007; Liu,C. et al., 2014; Liu, J. et al., 2014; Lu et al., 2011; Min et al., 2012; Shao et al., 2012; Wang et al., 2014; Wei et al., 2017; Wei et al., 1994; Wu et al., 2010; Xu et al., 2007; Zhang et al., 1999; Zhang et al., 2012; Zhang et al., 2011) and five (Hsing et al., 1994; Jian et al., 2004; Lee et al., 1998; Li et al., 2008; Yang et al., 2006) in English journals. Table 1 presents the basic characteristics of these studies including controlled covariates included in individual studies. As shown in Table 1, there were 36 risk estimates available for alcohol exposures across the 25 selected studies. These studies used 4,488 patients with diagnosis of incidence of prostate cancer as cases but different controls (a total of 7,830 controls) and defined the exposures differently. Eight OR estimates used hospital patients with diagnosis of benign prostatic hyperplasia as controls (Jia et al., 2013; Liang et al., 2007; Liu, C. et al., 2014; Liu, J. et al., 2014; Wu, 2008; Zhang et al., 2012), nine healthy subjects (Hsing et al., 1994; Lee et al., 1998; Li et al., 2008; Lu et al., 2011; Min et al., 2012; Shao et al., 2012; Wang et al., 2014; Wu, 2011; Zhang et al., 2011), 16 patients without a diagnosis of cancer (Hsing et al., 1994; Jian et al., 2004; Ma, 2014; Wei et al., 2017; Wu et al., 2010; Xu et al., 2007) or prostate cancer (Dong et al., 2016) or prostate disease (Jia et al., 2013; Liang et al., 2007; Liu, C. et al., 2014; Liu, J. et al., 2014; Wu, 2008; Zhang et al., 1999), and three patients with other cancer (Hsing et al., 1994) or other cancers (Wei et al., 1994) or other urological diseases (Yang et al., 2006).

\section{Table 2}

Definition of non-drinkers and drinkers in 25 Chinese case-controlled studies on alcohol use and prostate cancers

\begin{tabular}{|c|c|c|c|c|}
\hline Author & $\begin{array}{l}\text { Pub } \\
\text { year }\end{array}$ & OR $(95 \% \mathrm{CI})$ & Definition of abstainer reference & $\begin{array}{c}\text { Definition of alcohol } \\
\text { drinkers }\end{array}$ \\
\hline Hsing et al. & 1994 & $1.56(1.02-2.38)$ & Never alcohol use & Ever alcohol use \\
\hline Hsing et al. & 1994 & $0.67(0.40-1.11)$ & Never alcohol use & Ever alcohol use \\
\hline Hsing et al. & 1994 & $0.78(0.47-1.29)$ & Never alcohol use & Ever alcohol use \\
\hline Wei et al. & 1994 & $1.00(0.41-2.40)$ & No drink & Drink \\
\hline Lee et al. & 1998 & $1.53(0.99-2.36)$ & Never used alcohol & Ever used alcohol \\
\hline Zhang et al. & 1999 & $2.04(1.26-3.29)$ & No drink & Drink \\
\hline Jian et al. & 2004 & $1.29(0.78-2.11)$ & Never drank & $>0-25$ drinks $(1 \mathrm{D}=15 \mathrm{~g})$ weekly \\
\hline Jian et al. & 2004 & $1.22(0.72-2.06)$ & Never drank & $>25$ drinks $(1 \mathrm{D}=15 \mathrm{~g})$ weekly \\
\hline Yang et al. & 2006 & $1.15(0.76-1.74)$ & $<3$ times weekly for $10+$ years & $3+$ times weekly for $10+$ years \\
\hline Liang et al. & 2007 & $1.40(0.35-5.56)$ & $<1$ g per day & $1-100$ g per day \\
\hline Liang et al. & 2007 & $1.33(0.52-3.39)$ & $<1 \mathrm{~g}$ per day & $>100$ g per day \\
\hline Liang et al. & 2007 & $1.99(0.38-10.54)$ & $<1$ g per day & $1-100 \mathrm{~g}$ per day \\
\hline Liang et al. & 2007 & $2.17(0.71-6.64)$ & $<1$ g per day & $>100$ g per day \\
\hline $\mathrm{Xu}$ et al. & 2007 & $1.62(1.03-2.56)$ & $<3$ times weekly for $10+$ years & $3+$ times weekly for $10+$ years \\
\hline Li et al. & 2008 & $1.03(0.47-2.24)$ & Never drank & Ever drink \\
\hline Wu et al. & 2008 & $1.29(0.69-2.43)$ & $<1$ g per day & $1-100$ g per day \\
\hline Wu et al. & 2008 & $1.53(0.70-3.32)$ & $<1$ g per day & $>100$ g per day \\
\hline Wu et al. & 2008 & $1.71(0.86-3.40)$ & $<1 \mathrm{~g}$ per day & $1-100 \mathrm{~g}$ per day \\
\hline Wu et al. & 2008 & $1.53(0.75-3.10)$ & $<1 \mathrm{~g}$ per day & $>100$ g per day \\
\hline Wu et al. & 2010 & $0.82(0.57-1.18)$ & $<3$ times weekly for $10+$ years & $3+$ times weekly for $10+$ years \\
\hline $\mathrm{Xu}$ et al. & 2010 & $2.06(1.06-3.95)$ & $<1$ time weekly for $6+$ months & $1+$ time weekly for $6+$ months \\
\hline Lu et al. & 2011 & $0.80(0.56-1.16)$ & $<3$ times weekly for $10+$ years & $3+$ times weekly for $10+$ years \\
\hline Wu et al. & 2011 & $1.53(0.50-4.35)$ & $<1$ time weekly for $6+$ months & $1+$ time weekly for $6+$ months \\
\hline Zhang et al. & 2011 & $1.63(1.03-2.57)$ & $<1$ time weekly for $6+$ months & $1+$ time weekly for $6+$ months \\
\hline Min et al. & 2012 & $2.01(1.27-3.18)$ & No drink & Drink \\
\hline Shao et al. & 2012 & $1.84(1.19-2.84)$ & $<3$ times weekly for $10+$ years & $3+$ times weekly for $10+$ years \\
\hline Zhang et al. & 2012 & $2.46(1.02-5.91)$ & $<1$ time weekly for $6+$ months & $1+$ time weekly for $6+$ months \\
\hline Jia et al. & 2013 & $1.27(0.64-2.50)$ & $<150$ g per day & $150+$ g per day \\
\hline Jia et al. & 2013 & $1.94(0.94-4.01)$ & $<150$ g per day & $150+$ g per day \\
\hline Liu et al. & 2014 & $1.54(1.13-2.10)$ & Drink $<1$ year & Drink for $1+$ year \\
\hline Liu et al. & 2014 & $1.44(0.82-2.54)$ & No drink & Drink \\
\hline Liu et al. & 2014 & $1.58(0.89-2.80)$ & No drink & Drink \\
\hline Ma et al. & 2014 & $0.85(0.31-2.30)$ & Never drank & Ever drink \\
\hline Wang et al. & 2014 & $1.36(1.01-1.84)$ & $<3$ times weekly for $6+$ months & $3+$ times weekly for $6+$ months \\
\hline Dong et al. & 2016 & $1.30(1.02-1.65)$ & $<3$ times weekly for $6+$ months & $3+$ times weekly for $6+$ months \\
\hline Wei et al. & 2017 & $2.23(1.24-3.99)$ & Never drank & Ever drink \\
\hline
\end{tabular}

Note: The descriptions of the abstainers and drinkers are directly translated from the original studies. 
No studies examined dose-response relationship although three studies estimated ORs for two drinking categories (Jian et al., 2004; Liang et al., 2007; Wu, 2008). All these casecontrol studies presented the OR estimates of prostate cancer incidence for defined drinkers compared with defined nondrinkers. Defined drinkers are those who drank once a week for more than 6 months (4 OR estimates), at least 3 times a week for more than 6 months (2), at least 3 times a week for more than 10 years (5), drank for more than one year (2), $150+$ g per day (2), drank $1-25$ g per day (1) and $>25$ g per day (1), drank 1-100 g (4) and >100 g per day (4) and ever drank (11).

A forest plot (see Figure 2) displays the 36 OR estimates for the risk of prostate cancer associated with any level of drinking versus "abstaining" reported in individual studies. A visual inspection of Figure 2 indicates considerable crossstudy variation in estimates. Among 36 OR estimates for any drinking versus non-drinking in 25 studies, 12 OR estimates are significantly higher than one, and 18 higher and five lower than one but not statistically significant, and one equal one. Mean OR is 1.42 (95\% CI: $1.27-1.58, \mathrm{P}<0.0001)$ showing a significantly higher OR of prostate cancer among "drinkers" than "non-drinkers".

Table 3 presents unadjusted mean OR estimates of prostate cancer incidence overall and by study design (populationbased and hospital-based controls), drinker bias (non-drinker reference groups were not defined as never drank), types of drinkers, and studies with and without controlled smoking status with tests of publication bias and heterogeneity. Compared to the "non-drinkers", being a drinker was associated with a significantly increased risk of prostate cancer overall and by subgroups. Figure 3 provides a funnel plot showing the log-ORs and their inverse standard error from which there was no marked indication of publication bias as the plot is reasonably symmetrical. No significant publication bias was detected using the Egger's regression either for the pooled data or studies with different controls, drinker bias and defined drinking $(\mathrm{P}>0.05$ for each category). There was a significant heterogeneity detected using the Q statistic in either the pooled or some individual studies with different controls and defined drinking category estimates $(\mathrm{P}<0.01$ in each case $)$.

\section{Figure 3}

Funnel plot of relative risk (1n(OR)) of prostate cancer incidence due to alcohol consumption against inverse standard error of $\ln (\mathrm{OR}$ in 25 Chinese case-controlled studies

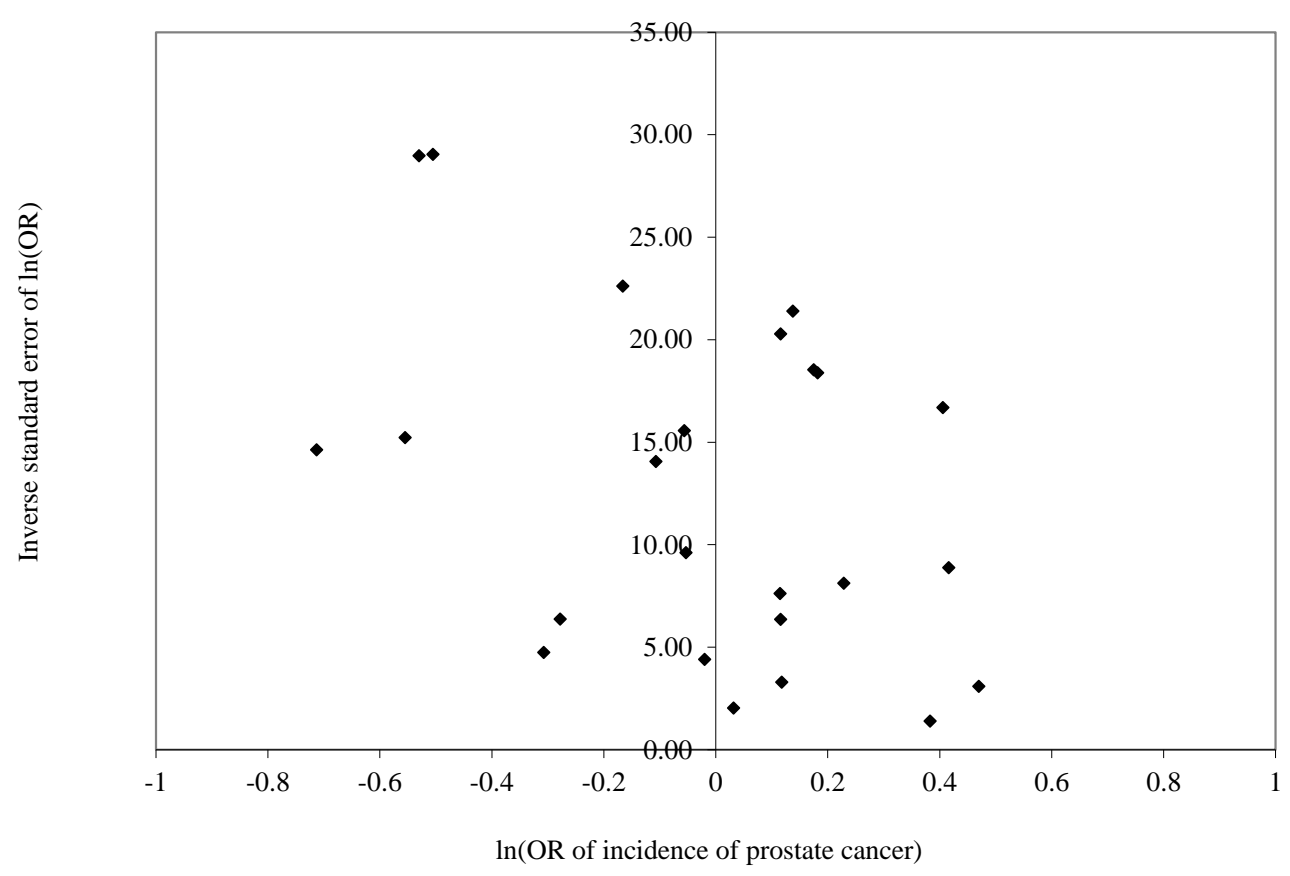


Table 3

Unadjusted mean odds ratio (OR) estimates of prostate cancer incidence and $95 \%$ confidence interval (CI) by studies with different controls, different definitions of drinkers for drinkers compared with 'abstainers' and controlled smoking status $(N=36)$ with tests of publication bias and heterogeneity

\begin{tabular}{|c|c|c|c|c|c|c|c|}
\hline \multirow[t]{2}{*}{ Subgroups } & \multirow[t]{2}{*}{$\mathbf{N} \dagger$} & \multicolumn{2}{|c|}{ Unadjusted mean OR } & \multicolumn{2}{|c|}{$\begin{array}{c}\text { Egger's regression for publication } \\
\text { bias }\end{array}$} & \multicolumn{2}{|c|}{ Test for heterogeneity } \\
\hline & & OR $(95 \%$ CI $)$ & T-test $p$ & Coefficient & T-test $p$ & Q statistic $p$ & $I^{2}(\%, 95 \% \mathrm{CI})$ \\
\hline Non-drinker & & 1.00 & & & & & \\
\hline \multicolumn{8}{|l|}{ Control definition } \\
\hline Healthy control & 9 & $1.43(1.15-1.77)$ & 0.0020 & +0.54 & 0.6063 & $<0.001$ & $44.67(00.00-74.41)$ \\
\hline T-test $p \dagger \dagger$ & & & 0.9377 & & & & \\
\hline \multicolumn{8}{|l|}{ Drinker bias $\ddagger$} \\
\hline No & 14 & $1.29(1.03-1.53)$ & 0.0040 & -1.11 & 0.2883 & $<0.001$ & $44.51(00.00-70.33)$ \\
\hline $\mathrm{T}$-test $p \dagger \dagger$ & & & 0.1566 & & & & \\
\hline \multicolumn{8}{|l|}{ Drinker definition $\$+$} \\
\hline $\begin{array}{l}\text { Heavy or drank for } 6+ \\
\text { months }\end{array}$ & 19 & $1.52(1.32-1.76)$ & 0.0001 & +2.10 & 0.0512 & $<0.001$ & $58.70(31.44-75.12)$ \\
\hline Ever drank & 17 & $1.31(1.13-1.53)$ & 0.0010 & -0.13 & 0.8991 & $<0.001$ & $49.05(10.74-70.92)$ \\
\hline $\mathrm{T}$-test $p \dagger \dagger$ & & & 0.1604 & & & & \\
\hline \multicolumn{8}{|l|}{ Controlled smoking $+\uparrow t$} \\
\hline No & 11 & $1.23(1.02-1.48)$ & 0.0313 & -0.31 & 0.7611 & $<0.001$ & $62.88(28.79-80.65)$ \\
\hline Yes & 25 & $1.51(1.33-1.71)$ & 0.0001 & +1.31 & 0.2041 & $<0.001$ & $54.63(28.73-71.12)$ \\
\hline $\mathrm{T}$-test $p \dagger \dagger$ & & & 0.0634 & & & & \\
\hline
\end{tabular}

$\dagger$ Number of OR estimates.

$\dagger \dagger$ Test difference in RR between two subgroups.

\$ Studies that defined "Never drank/no drink" as reference were specified as no drinker bias; otherwise drinker bias.

$+\$$ Heavy drink includes those studies that defined drinkers as those who drank at least one time for $6+$ months, at least three times for $6+$ months, at least three times for $10+$ years, drink for $1+$ year and $100+\mathrm{g}$ or $150+\mathrm{g}$ per day or drink $>25$ drinks per week.

Smoking factor was controlled or not in study designs or analysis. 
Table 4

Adjusted mean relative risk (RR) estimates of prostate cancer incidence by studies with different controls, reference group with and without former and occasional drinker bias and with different definitions of drinkers for drinkers compared with 'abstainers' $(N=36)$

\begin{tabular}{|c|c|c|c|c|c|c|c|}
\hline \multirow[t]{2}{*}{ Subgroups } & \multirow[t]{2}{*}{$\mathbf{N} \dagger$} & \multicolumn{2}{|c|}{$\begin{array}{l}\text { Mean RR corrected for } \\
\text { overestimated OR } \\
\text { (A) }\end{array}$} & \multicolumn{2}{|c|}{$\begin{array}{l}\text { (A) Weighted and adjusted for } \\
\text { between-study variation } \\
\text { (B) }\end{array}$} & \multicolumn{2}{|c|}{ 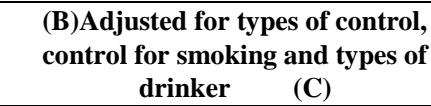 } \\
\hline & & RR (95\% CI) & T-test $p$ & RR (95\% CI) & T-test $p$ & RR $(95 \%$ CI $)$ & T-test $p$ \\
\hline Non-drinker & & 1.00 & & 1.00 & & 1.00 & \\
\hline \multicolumn{8}{|l|}{ Control definition } \\
\hline Hospital patients & 27 & $1.41(1.25-1.60)$ & 0.0001 & $1.31(1.12-1.565)$ & 0.0040 & $1.32(1.12-1.57)$ & 0.0048 \\
\hline Healthy control & 9 & $1.43(1.15-1.77)$ & 0.0020 & $1.62(1.30-2.02)$ & 0.0006 & $1.61(1.25-2.06)$ & 0.0020 \\
\hline T-test $p \dagger \dagger$ & & & 0.9377 & & 0.0928 & & 0.1495 \\
\hline \multicolumn{8}{|l|}{ Drinker bias $\ddagger$} \\
\hline No & 14 & $1.29(1.09-1.53)$ & 0.0040 & $1.42(1.15-1.76)$ & 0.0037 & $1.49(1.15-1.92)$ & 0.0071 \\
\hline Yes & 22 & $1.50(1.32-1.72)$ & 0.0001 & $1.38(1.17-1.62)$ & 0.0011 & $1.43(1.17-1.75)$ & 0.0032 \\
\hline T-test $p \dagger \dagger$ & & & 0.1566 & & 0.8080 & & 0.8031 \\
\hline \multicolumn{8}{|l|}{ Drinker definition $\$$} \\
\hline Heavy or drank for $6+$ months & 19 & $1.52(1.32-1.76)$ & 0.0001 & $1.42(1.20-1.68)$ & 0.0009 & $1.50(1.19-1.88)$ & 0.0032 \\
\hline Ever drank & 17 & $1.31(1.13-1.53)$ & 0.0010 & $1.36(1.13-1.65)$ & 0.0065 & $1.42(1.16-1.74)$ & 0.0036 \\
\hline T-test $p \dagger \dagger$ & & & 0.1604 & & 0.7103 & & 0.7036 \\
\hline \multicolumn{8}{|l|}{ Controlled smoking $+t \leftarrow$} \\
\hline No & 11 & $1.23(1.02-1.48)$ & 0.0313 & $1.30(1.07-1.59)$ & 0.0125 & $1.45(1.11-1.90)$ & 0.0112 \\
\hline Yes & 25 & $1.51(1.33-1.71)$ & 0.0001 & $1.46(1.24-1.72)$ & 0.0003 & $1.46(1.21-1.76)$ & 0.0013 \\
\hline T-test $p \dagger \dagger$ & & & 0.0694 & & 0.3522 & & 0.9779 \\
\hline Pooled all studies & 36 & $1.42(1.27-1.58)$ & 0.0001 & $1.40(1.24-1.58)$ & 0.0001 & $1.46(1.40-1.52)$ & 0.0001 \\
\hline
\end{tabular}

$\dagger \mathrm{N}=$ Number of RR estimates (correct effect of OR overestimated RR in (A), further adjusted for sampling variability and between-study variation in (B) and further adjusted for types of control, drinker bias, defined drinkers and controlled smoking status in $(\mathrm{C})$ ).

$\dagger \dagger$ test difference in RR between two subgroups.

Studies that defined "Never drank/no drink" as reference were specified as no drinker bias; otherwise drinker bias.

$+\$$ Heavy drink includes those studies that defined drinkers as those who drank at least one time for $6+$ months, at least three times for $6+$ months, at least three times for $10+$ years, drink for $1+$ year and $100+\mathrm{g}$ or $150+\mathrm{g}$ per day or drink $>25$ drinks per week.

+ Smoking factor was controlled or not in study designs or analysis. 
After correcting for the effect of OR overestimated RR (A Table 4), we next estimated the weighted RR further adjusted for between-study variation (B) and the weighted RRs adjusted for between-study variation were further adjusted for potential confounding effects of types of controls, drinker bias, definitions of drinkers and controlled smoking status (C). These analyses are presented in Table 4. After correcting the effect of OR overestimated RR, the mean $\mathrm{RR}$ of 36 risk estimates did not change, i.e., RR being still 1.42 (95\% CI: $1.27-1.58, \mathrm{P}<0.0001)$ because the low incidence rate in Chinese population; the mean RR estimates also remained unchanged for all subgroups (Column A in Table 4). The weighted mean RR of 36 risk estimates was 1.40 (95\% CI: $1.24-1.58, \mathrm{P}<0.0001$ ) after adjusting for the effect of between-study variation (Column B in Table 4). The fully adjusted RR was 1.46 (95\% CI: $1.40-1.52$,
$\mathrm{P}<0.0001)$ after further adjusting for the effects of types of controls, whether or not the studies controlled for smoking status, and how the studies defined reference group nondrinkers and drinkers in individual studies (Column $\mathrm{C}$ in Table 4). The fully-adjusted RRs for drinkers versus nondrinkers varied across subgroups but no significant differences were identified between subgroups.

Inclusion and/or exclusion for any study did not affect the mean risk estimate. After excluding of five articles or eight OR estimates published in English journals, the adjusted RR estimate remained unchanged. The sensitivity analyses of risk estimates are presented in Table 5.

\section{Table 5}

Sensitivity analysis of risk estimates of 25 case-control studies by exclusion one by one for analysis of the association of incidence risk of prostate cancer with alcohol consumption among Chinese people, 1994-2017

\begin{tabular}{|c|c|c|c|c|c|c|}
\hline Author & $\begin{array}{c}\text { Publication } \\
\text { year }\end{array}$ & $\mathrm{N}$ of ORs & $\begin{array}{c}\text { Unadjusted OR } \\
(95 \% \mathrm{CI}) \dagger\end{array}$ & T-test $p$ & $\begin{array}{c}\text { Fully-adjusted RR } \\
(95 \% \text { CI }) \ddagger\end{array}$ & T-test $p$ \\
\hline Hsing et al. & 1994 & 3 & $1.47(1.33-1.63)$ & 0.0001 & $1.40(1.36-1.45)$ & 0.0001 \\
\hline Wei et al. & 1994 & 1 & $1.43(1.28-1.60)$ & 0.0001 & $1.45(1.40-1.51)$ & 0.0001 \\
\hline Lee et al. & 1998 & 1 & $1.41(1.26-1.58)$ & 0.0001 & $1.48(1.40-1.57)$ & 0.0001 \\
\hline Zhang et al. & 1999 & 1 & $1.40(1.26-1.57)$ & 0.0001 & $1.40(1.34-1.47)$ & 0.0001 \\
\hline Jian et al. & 2004 & 2 & $1.43(1.27-1.60)$ & 0.0001 & $1.46(1.39-1.52)$ & 0.0001 \\
\hline Yang et al. & 2006 & 1 & $1.43(1.27-1.59)$ & 0.0001 & $1.48(1.41-1.56)$ & 0.0001 \\
\hline Liang et al. & 2007 & 4 & $1.39(1.23-1.56)$ & 0.0001 & $1.43(1.38-1.49)$ & 0.0001 \\
\hline Xu et al. & 2007 & 1 & $1.41(1.26-1.58)$ & 0.0001 & $1.44(1.38-1.51)$ & 0.0001 \\
\hline Li et al. & 2008 & 1 & $1.43(1.28-1.60)$ & 0.0001 & $1.51(1.42-1.61)$ & 0.0001 \\
\hline Wu et al. & 2008 & 4 & $1.41(1.24-1.59)$ & 0.0001 & $1.44(1.37-1.50)$ & 0.0001 \\
\hline Wu et al. & 2010 & 1 & $1.44(1.29-1.60)$ & 0.0001 & $1.46(1.42-1.51)$ & 0.0001 \\
\hline $\mathrm{Xu}$ et al. & 2010 & 1 & $1.40(1.26-1.57)$ & 0.0001 & $1.47(1.38-1.56)$ & 0.0001 \\
\hline Lu et al. & 2011 & 1 & $1.44(1.29-1.60)$ & 0.0001 & $1.50(1.42-1.58)$ & 0.0001 \\
\hline Wu et al. & 2011 & 1 & $1.41(1.26-1.58)$ & 0.0001 & $1.45(1.39-1.52)$ & 0.0001 \\
\hline Zhang et al. & 2011 & 1 & $1.41(1.26-1.58)$ & 0.0001 & $1.47(1.39-1.55)$ & 0.0001 \\
\hline Min et al. & 2012 & 1 & $1.40(1.26-1.57)$ & 0.0001 & $1.42(1.37-1.47)$ & 0.0001 \\
\hline Shao et al. & 2012 & 1 & $1.41(1.26-1.57)$ & 0.0001 & $1.44(1.38-1.50)$ & 0.0001 \\
\hline Zhang et al. & 2012 & 1 & $1.39(1.25-1.55)$ & 0.0001 & $1.48(1.38-1.59)$ & 0.0001 \\
\hline Jia et al. & 2013 & 2 & $1.41(1.26-1.58)$ & 0.0001 & $1.50(1.39-1.52)$ & 0.0001 \\
\hline Liu et al. & 2014 & 2 & $1.41(1.26-1.58)$ & 0.0001 & $1.44(1.37-1.52)$ & 0.0001 \\
\hline Liu et al. & 2014 & 1 & $1.41(1.26-1.58)$ & 0.0001 & $1.45(1.38-1.53)$ & 0.0001 \\
\hline Ma et al. & 2014 & 1 & $1.44(1.29-1.60)$ & 0.0001 & $1.48(1.41-1.55)$ & 0.0001 \\
\hline Wang et al. & 2014 & 1 & $1.42(1.27-1.59)$ & 0.0001 & $1.51(1.41-1.52)$ & 0.0001 \\
\hline Dong et al. & 2016 & 1 & $1.42(1.27-1.59)$ & 0.0001 & $1.48(1.40-1.57)$ & 0.0001 \\
\hline Wei et al. & 2017 & 1 & $1.40(1.25-1.56)$ & 0.0001 & $1.41(1.34-1.48)$ & 0.0001 \\
\hline Cancer control †† & 1994 & 2 & $1.46(1.32-1.62)$ & 0.0001 & $1.41(1.37-1.46)$ & 0.0001 \\
\hline Articles/Mandarin & 1994-2008 & 8 & $1.52(1.35-1.71)$ & 0.0001 & $1.48(1.41-1.55)$ & 0.0001 \\
\hline 36 risks pooled & 1994-2017 & 36 & $1.42(1.27-1.58)$ & 0.0001 & $1.46(1.40-1.52)$ & 0.0001 \\
\hline
\end{tabular}

$\uparrow$ Unadjusted OR by excluding each study.

I Fully-adjusted RR by excluding each study.

$\uparrow \dagger$ Two risk estimates used other cancer patients as controls Hsing et al. (1994) and Wei et al. (1994).

We conducted sensitivity analyses by including or excluding each of the studies in meta-regression in order to examine their effects.

The dose-response analysis showed that drinkers who drank 1-55 g per day, 56-100 or over 100 had a significantly higher risk of suffering from prostate cancer than healthy/patient controls (OR=1.51, 1.43 or 1.59). The OR significantly increased as drinking alcohol amount increased per day (Wald test $\mathrm{P}<0.001$ ). 
Table 6

Estimated odds ratio (OR) and 95\% confidence interval (CI) of prostate cancer for three drinking groups versus non-drinkers among Chinese people

\begin{tabular}{|c|c|c|c|c|c|c|c|c|c|c|c|c|c|c|c|c|}
\hline \multirow{2}{*}{ Ethanol grams per day } & \multicolumn{4}{|c|}{ Number of cases and controls $\dagger$} & \multicolumn{4}{|c|}{ Healthy people as control } & \multicolumn{4}{|c|}{ Patient as control } & \multicolumn{4}{|c|}{ Healthy/patient as control } \\
\hline & Cases & $\mathrm{HC}$ & DC & HP & OR & $95 \%$ & CI & $x^{2} P$ & OR & $95 \%$ & CI & $x^{2} P$ & OR & $95 \%$ & CI & $x^{2} P$ \\
\hline \multicolumn{17}{|l|}{ Jian et al. 2004} \\
\hline Non-drinker & 60 & 142 & & & 1.00 & & & & & & & & & & & \\
\hline $1-55 \mathrm{~g}$ & 38 & 70 & & & 1.28 & 0.78 & 2.11 & ns & & & & & & & & \\
\hline $56+\mathrm{g}$ & 32 & 62 & & & 1.22 & 0.72 & 2.06 & ns & & & & & & & & \\
\hline \multicolumn{17}{|l|}{ Liang et al. 2007} \\
\hline Non-drinker & 96 & 106 & 109 & 215 & 1.00 & & & & 1.00 & & & & 1.00 & & & \\
\hline $1-100$ & 25 & 21 & 17 & 38 & 1.31 & 0.69 & 2.50 & ns & 1.67 & 0.85 & 3.28 & $\mathrm{~ns}$ & 1.47 & 0.84 & 2.58 & $\mathrm{~ns}$ \\
\hline $101+$ & 21 & 15 & 16 & 31 & 1.55 & 0.75 & 3.17 & $\mathrm{~ns}$ & 1.49 & 0.74 & 3.02 & $\mathrm{~ns}$ & 1.03 & 0.49 & 2.18 & ns \\
\hline \multicolumn{17}{|l|}{ Wu et al. 2008} \\
\hline Non-drinker & 44 & 47 & 51 & 98 & 1.00 & & & & 1.00 & & & & 1.00 & & & \\
\hline $1-100$ & 6 & 5 & 5 & 10 & 1.28 & 0.37 & 4.50 & ns & 1.39 & 0.40 & 4.87 & $\mathrm{~ns}$ & 1.34 & 0.46 & 3.91 & $\mathrm{~ns}$ \\
\hline $101+$ & 12 & 10 & 6 & 16 & 1.28 & 0.50 & 3.26 & ns & 2.32 & 0.80 & 6.69 & ns & 1.25 & 0.35 & 4.40 & $\mathrm{~ns}$ \\
\hline \multicolumn{17}{|c|}{ Liang et al. 2007+Wu et al. 2008} \\
\hline Non-drinker & 140 & 153 & 160 & 313 & 1.00 & & & & 1.00 & & & & 1.00 & & & \\
\hline $1-100$ & 31 & 26 & 22 & 48 & 1.30 & 0.74 & 2.30 & ns & 1.61 & 0.89 & 2.91 & ns & 1.44 & 0.88 & 2.37 & ns \\
\hline $101+$ & 33 & 25 & 22 & 47 & 1.44 & 0.82 & 2.55 & ns & 1.71 & 0.95 & 3.08 & ns & 1.09 & 0.58 & 2.05 & $\mathrm{~ns}$ \\
\hline \multicolumn{17}{|l|}{ All 25 included studies } \\
\hline Non-drinker & 3,087 & 2,450 & 3,671 & 6,122 & 1.00 & & & & 1.00 & & & & 1.00 & & & \\
\hline $1-55 \mathrm{~g}$ & 371 & 221 & 268 & 489 & 1.33 & 1.12 & 1.59 & $* *$ & 1.65 & 1.40 & 1.94 & $* * *$ & 1.51 & 1.31 & 1.74 & $* * *$ \\
\hline $56-100 \mathrm{~g}$ & 312 & 196 & 237 & 433 & 1.26 & 1.05 & 1.53 & $*$ & 1.57 & 1.32 & 1.87 & $* * *$ & 1.43 & 1.23 & 1.67 & $* * *$ \\
\hline $101+$ & 728 & 400 & 505 & 905 & 1.44 & 1.26 & 1.65 & $* * *$ & 1.71 & 1.52 & 1.94 & $* * *$ & 1.59 & 1.43 & 1.77 & $* * *$ \\
\hline Drinker & 1,411 & 817 & 1,010 & 1,826 & 1.37 & 1.24 & 1.52 & $* * *$ & 1.66 & 1.51 & 1.83 & $* * *$ & 1.53 & 1.41 & 1.66 & $* * *$ \\
\hline
\end{tabular}

$\dagger \mathrm{HC}=$ healthy people as control, $\mathrm{DC}=$ disease people as controls and $\mathrm{HP}=$ combined healthy and patient controls. Wald test: ns $\mathrm{P}>0.05 * \mathrm{P}<0.05 * * \mathrm{P}<0.01 * * * \mathrm{P}<0.001$. 


\section{Discussion}

We present a meta-regression analysis of the case-control studies examining the relationship between incidence of prostate cancer and alcohol consumption in Chinese population. The study included 25 studies with 36 OR estimates to produce mean RR of prostate cancer due to alcohol use.

The analysis shows a $46 \%$ significantly increased RR of prostate cancer for drinkers versus non-drinkers (RR: 1.46, 95\% CI: $1.40-1.52$ ) after adjusting for the effects of overestimated RR using ORs in case-control studies, sampling variability and between-study variation and the effects of using different controls, controlled smoking status and differently defining drinkers/non-drinkers in the individual studies. The dose-response analysis showed that the risk of prostate cancer significantly increased as drinking alcohol amount increased per day. These risk estimates are consistent with that estimated in other published metaanalysis (Zhao et al., 2016) but markedly higher in this study than that one ( $R R=1.46$ vs 1.08$)$.

Among 36 RR estimates in 25 studies, 9 used "healthy people" as controls and 27 used patients as controls. The RR estimate using healthy people as controls had a markedly higher RR than that using patients as controls (1.61 vs 1.32). This was because alcohol consumption caused many diseases (Rehm, Gmel, Sempos, \& Trevisan, 2003) and thus using patients as controls could underestimate the RR of prostate cancer. Further research should avoid using patients as controls.

Sixteen of the 25 included studies have clearly misclassified former drinkers and occasional or light drinkers into the lifetime non-drinkers. The other nine studies specified nondrinker reference groups as "never drank or no drink". Studies have shown that including former and occasional drinkers in the non-drinking reference or comparisons group tends to underestimate the risk of prostate cancer for drinkers (Shaper, Wannamethee, \& Walker, 1988; Stockwell et al., 2016). The meta-analysis on prostate cancer and alcohol use by Zhao et al. (2016) showed an $8 \%$ increase in the RR of prostate cancer of any drinking versus non-drinking in the studies without former and occasional drinker biases compared with that in the studies with both former and occasional drinker biases. Our analysis showed a 6\% difference in fully adjusted RR estimates among Chinese population.

The adjusted mean of 19 RR estimates in 15 studies that defined drinkers as those who drank at least one time for $6+$ months, at least three times for $6+$ months, at least three times for $10+$ years, drink for $1+$ year and $100+\mathrm{g}$ or $150+\mathrm{g}$ per day or drink $>25$ drinks per week was higher than that of other 17 RR estimates. This suggested that heavy drinkers had a higher risk of prostate cancer incidence.

Alcohol is a known carcinogen causing a variety of human cancers (World Cancer Research Fund/American Institute for Cancer Research, 2007) via different biological pathways depending on the anatomical site. The evidence that alcoholic drinks are a cause of cancers of the mouth, pharynx, larynx, oesophagus, liver, colorectum and breast in women is compelling (Bagnardi et al., 2001; English, Holman, D'Arcy, \& Australia Department of Community Services and Health National Drug Strategy, 1995; World Cancer Research Fund/American Institute for Cancer Research, 2007). Alcoholic beverages are multicomponent mixtures containing several carcinogenic compounds such as ethanol, acetaldehyde, aflatoxins and ethyl carbamate (Lachenmeier, Przybylski, \& Rehm, 2012) and all of these compounds may contribute to increase the risk of cancer due to alcohol consumption reported in observational studies. The biological mechanisms by which alcohol intake might increase the risk of prostate cancer are not fully understood but the main mechanisms are likely to include a genotoxic effect of acetaldehyde, the induction of microsomal cytochrome P450 2E1 (CYP2E1) and associated oxidative stress, increased estrogen concentration, a role as a solvent for tobacco carcinogens, changes in folate metabolism, and changes in DNA repair (Baan et al., 2007; Homann et al., 2006; International Agency for Research on Cancer [IARC] 2010).

There are several limitations with this meta-analysis. Our meta-analysis was based on 25 studies including 36 risk estimates. This sample is relatively small when conducting multivariate meta-regression to control for study level characteristics that might confound the relationship between prostate cancer and alcohol consumption. While there were relatively few studies available for this analysis, the required number of observations per variable for linear regression is much smaller than in logistic regression. According to Austin and Steyerberg (2015) even two subjects per variable would not bias an estimate made using linear regression analysis (Austin \& Steyerberg, 2015). Furthermore, adjustment for study level characteristics such as whether smoking status was controlled is of course not as precise as controlling for this variable at the individual level within a study. Uncontrolled confounding from unmeasured or imprecisely measured variables will be present both within and between studies. The great majority of identified studies suffered one or more serious methodological problems including the widespread practice of misclassifying former drinkers as abstainers. This study identified only 25 casecontrol studies which 22 of these studies investigated the risk of prostate cancer among Chinese drinkers versus nondrinkers defined by the authors and the dose-response relationship cannot be examined using all 25 included studies. There were three studies that classified subjects into three levels of drinking. The cases and controls can be distributed into categories of non-drinkers, drinkers who drank 1-55 g, 56-100 g and more than $100 \mathrm{~g}$ per day according to the distributions of cases and controls in these three studies. We assumed that cases and controls had the same distributions of non-drinkers and drinkers in each of 25 studies and thus estimated the number of non-drinkers and drinker in three drinking groups. The analysis found that the OR significantly increased as drinking alcohol amount increased per day. 
The measures of alcohol consumption in these studies are mostly imprecise, including nine studies that specified "never drank" or "no drinking" as the reference group and thus may largely have confounded the risk estimates. However, it was expected that all the studies underestimated risk estimates using the definitions of drinkers versus nondrinkers and the present risk estimates can be considered conservative. This study was unable to incorporate the recommendation from Liang and Chikritzhs (2013) that former drinkers should in fact be included within the category of current drinkers according to previous drinking level so as to create an unbiased estimate of the risk relationship. For example, Hayes and colleagues (1996) found that among both blacks and whites, the risk associated with alcohol use was similarly increased among both recent (current drinker or quit in the last year) and former consumers of alcohol.

In summary, there is a significantly higher risk of prostate cancer incidence among Chinese drinkers than non-drinkers. The estimated risk is markedly higher in Chinese population than in the Caucasian population. Few Chinese studies examined the dose-response relationship and no cohort studies were identified in Chinese databases and in previously published reviews and meta-analyses. Future case-control and cohort studies should consider these issues which may have affected the estimated risk of prostate cancer due to alcohol consumption and investigate the doseresponse relationship in the Chinese population. We recommend that future studies on alcohol and disease seek to avoid biasing risk estimates by misclassifying either (i) former or occasional drinkers as abstainers, or (ii) occasional drinkers as low volume drinkers. We also recommend that former drinkers are classified with drinkers according to their past level of consumption.

\section{Declarations}

Ethics approval and consent to participate: Research ethics committee review and approval were not required, as aggregate data were extracted from published studies. Authors of some studies were contacted to clarify relevant information in the studies but no information on individuals in the studies was requested.

Role of the funding sources: The study funders had no role in study design, data collection, analysis or interpretation, report preparation and the decision to publish. All authors had full access to all the data and had final responsibility for the decision to submit for publication.

Consent for publication: Not applicable.

Availability of data and material: All data generated and analyzed during this study are included in this published article and can be found in Appendix A: Meta-analysis data on prostate cancer and alcohol consumption in Chinese population.

Competing Interests: None.
Authors Contribution: All authors made contributions to conception and design of the study. DG and YL searched studies from five Chinese databases and created the dataset and JZ searched five studies in English and added them to the dataset. JZ and TS checked all the included studies and coding. JZ and DG analyzed the data and drafted the manuscript. All authors revised the manuscript critically for intellectual content and give final approval of the version to be submitted.

Acknowledgement: We thank the authors of included studies who provided the data necessary for our metaanalysis.

\section{References}

Austin, P. C., \& Steyerberg, E. W. (2015). The number of subjects per variable required in linear regression analyses. Journal of Clinical Epidemiology, 68(6), 627636. doi: 10.1016/j.jclinepi.2014.12.014

Baade, P. D., Youlden, D. R., Cramb, S. M., Dunn, J., \& Gardiner, R. A. (2013). Epidemiology of prostate cancer in the Asia-Pacific region. Prostate International, 1(2), 47-58. doi: 10.12954/PI.12014

Baade, P. D., Youlden, D. R., \& Krnjacki, L. J. (2009). International epidemiology of prostate cancer: Geographical distribution and secular trends. Molecular Nutrition \& Food Research, 53(2), 171-184. doi: 10.1002/mnfr.200700511

Baan, R., Straif, K., Grosse, Y., Secretan, B., El Ghissassi, F., Bouvard, V., . . . Cogliano, V (2007). Carcinogenicity of alcoholic beverages. Lancet Oncology, 8(4), 292-293. doi: 10.1016/S1470-2045(07)70099-2

Bagnardi, V., Blangiardo, M., La Vecchia, C., \& Corrao, G. (2001). A meta-analysis of alcohol drinking and cancer risk. British Journal of Cancer, 85(11), 1700-1705. doi: 10.1054/bjoc.2001.2140

Bostwick, D. G., Crawford, D., E., Higano, C. S., \& Roach III, M. (2004). American Cancer Society's complete guide to prostate cancer. Atlanta, GA: American Cancer Society.

Breslow, R. A., \& Weed, D. L. (1998). Review of epidemiologic studies of alcohol and prostate cancer: 1971-1996. Nutrition and Cancer, 30(1), 1-13.

Chen, W. Q., Zheng, R. S., Baade, P. D., Zhang, S. W., Zeng, H. M., Bray, F., . . . He, J. (2016). Cancer statistics in China, 2015. CA: A Cancer Journal for Clinicians, 66(2), 115-132. doi: 10.3322/caac.21338

Dagnelie, P. C., Schuurman, A. G., Goldbohm, R. A., \& Van den Brandt, P. A. (2004). Diet, anthropometric measures and prostate cancer risk: A review of prospective cohort and intervention studies. BJU International, 93(8), 1139-1150. doi: 10.1111/j.1464-410X.2004.04795.x

Dennis, L. K. (2000). Meta-analysis for combining relative risks of alcohol consumption and prostate cancer. Prostate, 42(1), 56-66.

Dong, Y., Zhang, L., Zhang, Q., Wang, Q., Fan, S., Zhang, Z., . . Ju, X. (2016). Genetic polymorphisms in IGF-1 and IGFBP-3 are associated with prostate cancer in the Chinese population (IGF-1和IGFBP3 基因多态性与前列腺癌易感性的相关性). Chinese 
Journal of Surgical Oncology (中国肿瘤外科杂志), 8(2), 12.

Druesne-Pecollo, N., Tehard, B., Mallet, Y., Gerber, M., Norat, T., Hercberg, S., \& Latino-Martel, P. (2009). Alcohol and genetic polymorphisms: Effect on risk of alcohol-related cancer. Lancet Oncology, 10(2), 173180. doi: 10.1016/S1470-2045(09)70019-1

Edenberg, H. J. (2007). The genetics of alcohol metabolism: Role of alcohol dehydrogenase and aldehyde dehydrogenase variants. Alcohol Research \& Health, 30(1), 5-13.

English, D. R., Holman, C., D'Arcy, J., \& Australia Department of Community Services and Health National Drug Strategy. (1995). The quantification of drug caused morbidity and mortality in Australia / prepared for the Department of Human Services and Health for use in association with the National Drug Strategy. Canberra, Australia: Australia Government Publication Service.

Fillmore, K. M., Chikritzhs, T., Stockwell, T., Bostrom, A., \& Pascal, R. (2009). Alcohol use and prostate cancer: A meta-analysis. Molecular Nutrition \& Food Research, 53(2), 240-255. doi: 10.1002/mnfr.200800122

Forman, D., \& Ferlay, J. . (2014). The global and regional burden of cancer. In B. W. Stewart \& C. P. Wild (Eds.), World Cancer Report 2014 (pp. 16-53). Lyon, France: International Agency for Research on Cancer (IARC).

Gann, P. H. (2002). Risk factors for prostate cancer. Reviews in Urology, 4(Suppl 5), S3-S10.

Goedde, H. W., Agarwal, D. P., Fritze, G., Meiertackmann, D., Singh, S., Beckmann, G., . . . Czeizel, A. (1992). Distribution of $\mathrm{ADH} 2$ and $\mathrm{ALDH} 2$ genotypes in different populations. Human Genetics, 88(3), 344-346.

Hayes, R. B., Brown, L. M., Schoenberg, J. B., Greenberg, R. S., Silverman, D. T., Schwartz, A. G., . . . Pottern, L. M. (1996). Alcohol use and prostate cancer risk in US blacks and whites. American Journal of Epidemiology, 143(7), 692-697.

Hiatt, R. A., Armstrong, M. A., Klatsky, A. L., \& Sidney, S. (1994). Alcohol consumption, smoking, and other riskfactors and prostate cancer in a large health plan cohort in California (United States). Cancer Causes \& Control, 5(1), 66-72. doi: 10.1007/Bf01830728

Homann, N., Stickel, F., Konig, I. R., Jacobs, A., Junghanns, K., Benesova, M., . . . Seitz, H. K. (2006). Alcohol dehydrogenase $1 \mathrm{C}^{*} 1$ allele is a genetic marker for alcohol-associated cancer in heavy drinkers. International Journal of Cancer, 118(8), 1998-2002. doi: $10.1002 / \mathrm{Ijc} .21583$

Hsing, A. W., \& Devesa, S. S. (2001). Trends and patterns of prostate cancer: What do they suggest? Epidemiologic Reviews, 23(1), 3-13. doi: 10.1093/oxfordjournals.epirev.a000792

Hsing, A. W., Wang, R. T., Gu, F. L., Lee, M., Wang, T., Leng, T. J., . . . Blot, W. J. (1994). Vasectomy and prostate cancer risk in China. Cancer Epidemiology Biomarkers \& Prevention, 3(4), 285-288.

IARC (2010). International Agency for Research on Cancer (IARC) monographs on the evaluation of carcinogenic risks to humans: alcohol consumption and ethyl carbamate (Vol. 96). Lyon, France: IARC.

Ito, K. (2014). Prostate cancer in Asian men. Nature Reviews Urology, 11(4), 197-212. doi: 10.1038/nrurol.2014.42
Jia, Y., Sun, X., Gao, J., Zhu, L., Weng, B., Liu, Z., . . . Hou, S. (2013). Risk factors of prostate cancer in urban Qingdao: A case-control study (青岛市区前列腺癌发病危险因素的病例对照研究). National Journal of Andrology (中华男科学杂志), 19(8), 5.

Jian, L., Xie, L. P., Lee, A. H., \& Binns, C. W. (2004). Protective effect of green tea against prostate cancer: A case-control study in southeast China. International Journal of Cancer, 108(1), 130-135. doi: $10.1002 / \mathrm{ijc} .11550$

Kanda, J., Matsuo, K., Suzuki, T., Kawase, T., Hiraki, A., Watanabe, M., ... Tanaka, H. (2009). Impact of alcohol consumption with polymorphisms in alcoholmetabolizing enzymes on pancreatic cancer risk in Japanese. Cancer Science, 100(2), 296-302. doi: 10.1111/j.1349-7006.2008.01044.x

Lachenmeier, D. W., Przybylski, M. C., \& Rehm, J. (2012). Comparative risk assessment of carcinogens in alcoholic beverages using the margin of exposure approach. International Journal of Cancer, 131(6), E995-E1003. doi: $10.1002 / \mathrm{Ijc} .27553$

Lee, M. M., Wang, R. T., Hsing, A. W., Gu, F. L., Wang, T., \& Spitz, M. (1998). Case-control study of diet and prostate cancer in China. Cancer Causes \& Control, 9(6), 545-552. doi: 10.1023/A:1008840105531

Li, X. M., Li, J., Tsuji, I., Nakaya, N., Nishino, Y., \& Zhao, X. J. (2008). Mass screening-based case-control study of diet and prostate cancer in Changchun, China. Asian Journal of Andrology, 10(4), 551-560. doi: 10.1111/j.1745-7262.2008.00384.x

Li, Y., Yang, H. A., \& Cao, J. (2011). Association between alcohol consumption and cancers in the Chinese population - A systematic review and meta-analysis. Plos One, 6(4). doi: 10.1371/journal.pone.0018776

Liang, C., Liu, Q., Zhou, F., Gao, X., \& Chen, L. (2007). Case-control study on association between prostate cancer and smoking, drinking and physical activity (前列腺癌与生活行为方式病例对照研究). Chinese Journal of Public Health (中国公共卫生), 23(8), 3.

Liang, W. B., \& Chikritzhs, T. (2013). The association between alcohol exposure and self-reported health status: The effect of separating former and current drinkers. Plos One, 8(2). doi: 10.1371/journal.pone.0055881

Liu, C., Wang, Y., Gu, X., \& Zhou, G. (2014). A casecontrol study on association between prostate cancer and life behavior and sexuality (生活行为及性生活与前列腺癌危险性关系的病例对 照研究). Practical Geriatrics (实用老年医学), 28(11), 3.

Liu, J., Chen, Z., Bo, R., Dong, Y., Li, P., \& Yang, W. (2014). Case control studies of the relevant factors among Ningxia Hui and Han prostate cancer groups (宁夏回族自治区的回族及汉族前列腺癌患病因素的 病例对照研究). Chinese Journal of Preventive Medicine (中华预防医学杂志), 48(12), 5.

Longnecker, M. P. (1995). Alcohol consumption and risk of cancer in humans: An overview. Alcohol, 12(2), 87-96. doi: 10.1016/0741-8329(94)00088-3 
Lu, X., Hua, L., \& Wang, J. (2011). Association of IL-6 572C $>\mathrm{G}$ polymorphism with the susceptibility to prostate cancer in the Chinese Han population in Jiangsu and Anhui area (苏皖地区汉族人群IL-6$572 \mathrm{C}>\mathrm{G}$ 多态性与前列腺癌易感性的关系). National Journal of Andrology (中华男科学杂志), 17(8), 707711.

Ma, C. (2014). Association between single nucleotide polymorphisms on chromosome $17 q$ and the risk of prostate cancer in a Chinese population (单核甘酸多态性与广州汉族人群前列腺癌的相关性 研究). (PhD), Southern Medical University, Guangzhou, China. (R737.25)

Manthey, J., Shield, K. D., Rylett, M., Hasan, O. S. M., Probst, C., \& Rehm, J. (2019). Global alcohol exposure between 1990 and 2017 and forecasts until 2030: A modelling study. Lancet, 393(10190), 2493-2502. doi: 10.1016/S0140-6736(18)32744-2

Min, Z., Mi, Y., Shao, N., Feng, N., \& Hua, L. (2012). Association between CD14-260 polymorphism and the risk of the prostate cancer (CD14基因$260 \mathrm{~A} / \mathrm{G}$ 位点多态性与前列腺癌易感性的研究).

Journal of Modern Urology (现代泌尿外科杂志), $17(2), 4$.

Moher, D., Liberati, A., Tetzlaff, J., Altman, D. G., \& PRISMA Group. (2009). Preferred Reporting Items for Systematic Reviews and Meta-Analyses: The PRISMA Statement. Plos Medicine, 6(7). doi: ARTN e1000097DOI 10.1371/journal.pmed.1000097

Morton, M. S., Griffiths, K., \& Blacklock, N. (1996). The preventive role of diet in prostatic disease. British Journal of Urology, 77(4), 481-493. doi: 10.1046/j.1464-410X.1996.09361.x

National Health Commission of the People's Republic of China. (2019). Chinese guidelines for diagnosis and treatment of prostate cancer 2018 (English version). Chinese Journal of Cancer Research, 31(1), 7.

Rehm, J., Gmel, G., Sempos, C. T., \& Trevisan, M. (2003). Alcohol-related morbidity and mortality. Alcohol Research \& Health, 27(1), 39-51.

Rota, M., Scotti, L., Turati, F., Tramacere, I., Islami, F., Bellocco, R., . . . Bagnardi, V. (2012). Alcohol consumption and prostate cancer risk: A meta-analysis of the dose-risk relation. European Journal of Cancer Prevention, 21(4), 350-359. doi: 10.1097/Cej.0b013e32834dbc11

Sesso, H. D., Paffenbarger, R. S., \& Lee, I. M. (2001). Alcohol consumption and risk of prostate cancer: The Harvard Alumni Health Study. International Journal of Epidemiology, 30(4), 749-755. doi: 10.1093/Ije/30.4.749

Shao, N., Mi, Y., Min, Z., Feng, N., Zhang, W., \& Hua, L. (2012). Associations between-1304 T $>\mathrm{G}$ polymorphism in the promoter of mitogen-activated protein kinase kinase 4 gene and prostate cancer susceptibility (前列腺癌易感性与丝裂原活化蛋白激酶激酶4基因 多态性的关系). Chinese Journal of Experimental Surgery (中华实验外科杂志), 29(6), 4.

Shaper, A. G., Wannamethee, G., \& Walker, M. (1988). Alcohol and mortality in British men: Explaining the Ushaped curve. Lancet, 2(8623), 1267-1273.
Stemmermann, G. N., Nomura, A. M. Y., Chyou, P. H., \& Yoshizawa, C. (1990). Prospective-study of alcohol intake and large-bowel cancer. Digestive Diseases and Sciences, 35(11), 1414-1420. doi: 10.1007/Bf01536750

Stockwell, T., Zhao, J. H., Panwar, S., Roemer, A., Naimi, T., \& Chikritzhs, T. (2016). Do "moderate" drinkers have reduced mortality risk? A systematic review and meta-analysis of alcohol consumption and all-cause mortality. Journal of Studies on Alcohol and Drugs, 77(2), 185-198. doi: 10.15288/jsad.2016.77.185

Taitt, H. E. (2018). Global trends and prostate cancer: A review of incidence, detection, and mortality as influenced by race, ethnicity, and geographic location. American Journal of Mens Health, 12(6), 1807-1823. doi: 10.1177/1557988318798279

Tavani, A., Negri, E., Franceschi, S., Talamini, R., \& Lavecchia, C. (1994). Alcohol consumption and risk of prostate cancer. Nutrition and Cancer, 21(1), 25-31.

United Nations Department of Economic and Social Affairs Population Division. (2017). World Population Prospects: The 2017 Revision, Volume II: Demographic Profiles (ST/ESA/SER.A/400).

Vandergulden, J. W. J., Verbeek, A. L. M., \& Kolk, J. J. (1994). Smoking and drinking habits in relation to prostate cancer. British Journal of Urology, 73(4), 382389.

Wang, X., Chen, J., Qin, C., Shao, P., Wang, M., Zhang, Z., ... Yin, C. (2014). Association between VHL single nucleotide polymorphism (rs779805) and the susceptibility to prostate cancer (VHL基因多态性与前列腺癌易感性的相关性研究). China Journal of Modern Medicine (中国现代医学杂志), 24(3), 6.

Watters, J. L., Park, Y., Hollenbeck, A., Schatzkin, A., \& Albanes, D. (2010). Alcoholic beverages and prostate cancer in a prospective US cohort study. American Journal of Epidemiology, 172(7), 773-780. doi: 10.1093/Aje/Kwq200

Wei, G., Bin, X., \& Huang, Y. (2017). C Y P 17 基因多态性和环境暴露因素交互作用与前列腺癌易 感性的关系. Chinese Journal of Gerontology ( 中国老年学杂志), 37(5), 3 .

Wei, Q., Tang, X., Yang, Y., Zhan, Y., \& Yin, H. (1994). Risk factors of prostate cancer - a matched case-control study (前列腺癌发病危险因素的配比病例对照研究). Hua Xi Yi Ke Da Xue Xue Bao (华西医科大学学报), 25(1), 87-90.

WHO. (2010). International statistical classification of diseases and related health problems. Geneva: WHO. Retrieved from https://icd.who.int/browse10/2010/en. Accessed 30 August 2020.

Woodward, M. (2000). Epidemiology: Study design and data analysis (pp. 673-720). Boca Raton: Chapman \& Hall/CRC.

World Cancer Research Fund/American Institute for Cancer Research. (2007). Food, nutrition, physical activity, and the prevention of cancer: A global perspective. Washington DC: AICR.

Wu, H., Xu, B., \& Li, J. (2010). Correlation between P53 codon 72 polymorphism and prostate cancer risk in Han nationality population in Jiangsu and Anhui province 
(苏皖地区汉族人群P53

codon 72 多态性与前列腺癌易感性的关系). Chinese Journal of Andrology (中华男科学杂志), 24(5), 5.

$\mathrm{Wu}, \mathrm{Q}$. (2011). The screening study of PSA and case-control study of susceptible factors for prostate cancer in Shanghai,

China (前列腺癌PSA篣查及易患因素研究). (Master's thesis), Shanghai Jiao Tong University, Shanghai. (R737.25 R446.6)

Wu, Y. (2008). A case-control study of environmental and genetic factor of prostate cancer (前列腺癌环境和遗传因素的病例对照研究).

(Master's thesis), Sun Yat-sen University Guangzhou, China. (20080606)

Xia, J., Wright, J., \& Adams, C. E. (2008). Five large Chinese biomedical bibliographic databases: Accessibility and coverage. Health Information and Libraries Journal, 25(1), 55-61. doi: 10.1111/j.14711842.2007.00734.x

$\mathrm{Xu}$, $\mathrm{X}$. (2010). 中国人前列腺癌环境和遗传危险因素及基因甲基化 研究. (Master's thesis), Second Military Medical University, Shanghai, China. (R737.25)

Xu, Z., Qian, L., Hua, L., Wang, X., Yang, J., Zhang, W., \& $\mathrm{Wu}, \mathrm{H}$. (2007). Relationship between DNA repair gene XRCC1 Arg399Gln polymorphism and susceptibility to prostate cancer in the Han Population in Jiangsu and Anhui （苏 、皖地区汉族人群 DNA修复基因 XRCC1A rg399G $\ln$ 多态性与前列腺癌易感性的关系). National Journal of Andrology (中华男科学杂志), 13(4), 327-331.

Yang, J. (2006). Polymorphisms of metabolic enzyme genes, living habits and prostate cancer susceptibility : a case-control study in population of Han nationality in Southern China (中国南方汉族人群代谢酶基因多态性、生活习惯与 前列腺癌易感性及交互作用的研究).

(Master's thesis), Nanjing Medical University. (20060420)

Yang, J., Gu, M., Song, N., Feng, N., Hua, L., Ju, X., . . . $\mathrm{Wu}, \mathrm{H}$. (2009). Correlation of prostate cancer susceptibility with genetic polymorphism of cytochrome P450 2E1, smoking and drinking: a case-control study in the population of Nanjing Area (南京地区人群CYP2E1基因多态性及

吸烟、饮酒与前列腺癌易感性). National Journal of Andrology (中华男科学杂), 15(1), 7-11.

Yang, J., Qian, L. X., Wu, H. F., Xu, Z. Q., Sui, Y. G., Wang, X. R., \& Zhang, W. (2006). Genetic polymorphisms in the cytochrome P450 1A1 and 2E1 genes, smoking, drinking and prostate cancer susceptibility: A casecontrol study in a Han nationality population in Southern China. International Journal of Urology, 13(6), 773780. doi: 10.1111/j.1442-2042.2006.01401.x

Ye, D., \& Zhu, Y. (2015). Epidemiology of prostate cancer in China: An overview and clinical implication. Zhonghua Wai Ke Za Zhi [Chinese Journal of Surgery], 53(4), 249-252.

Zhang, H., Qiu, C., Shi, L., Liu, X., \& Chou, Y. (1999). Case-control study of risk factors of prostate cancer (前列腺癌危险因素配比病例对照研究).Journal Of
Preventive Medicine Information (预防医学情报杂志), 15(1), 2.

Zhang, L., Liang, Y., \& Cui, F. (2012). Methylation status in promoter of APC and environmental factors with the predisposition to prostate cancer (APC基因启动子区甲基化及环境因素与前列腺癌关 系研究). Shandong Medical Journal (山东医药), $52(38), 1-4$.

Zhang, L., Xu, X., Cui, X., Wang, G., Hou, J., Cao, G., . . Cui, F. (2011). Relationship of kallikrein 3 and vitamin D receptor polymorphisms and environmental factors with prostate cancer predisposition. Academic Journal of Second Military Medical University (第二军医大学学), 32(12), 1310-1315.

Zhao, J., Stockwell, T., Roemer, A., \& Chikritzhs, T. (2016). Is alcohol consumption a risk factor for prostate cancer? A systematic review and meta-analysis. BMC Cancer, 16(1), 845. doi: 10.1186/s12885-016-2891-Z

Zhao, J., Stockwell, T., Roemer, A., Naimi, T., \& Chikritzhs, T. (2017). Alcohol consumption and mortality from coronary heart disease: An updated meta-analysis of cohort studies. Journal of Studies on Alcohol and Drugs, 78(3), 375-386. doi: 10.15288/jsad.2017.78.375

Zintzaras, E. (2006). Methylenetetrahydrofolate reductase gene and susceptibility to breast cancer: A metaanalysis. Clinical Genetics, 69(4), 327-336. doi: 10.1111/j.1399-0004.2006.00605.x

Zintzaras, E., Stefanidis, L., Santos, M., \& Vidal, F. (2006). Do alcohol-metabolizing enzyme gene polymorphisms increase the risk of alcoholism and alcoholic liver disease? Hepatology, 43(2), 352-361. doi: 10.1002/hep. 21023 